\title{
Fortalecimiento de la conducta pro-ambientales en la IED Luz Marina Caballero ${ }^{1}$
}

\section{Strengthening pro-environmental behavior in IED Luz Marina Caballero}

DOI: http://dx.doi.org/10.17981/cultedusoc.9.3.2018.37

Artículo de investigación. Fecha de recepción: 15/06/2018. Fecha de aceptación: 27/11/2018

\author{
Heider De León-Sanabria²; \\ Luis Jiménez; Norys Cervantes; Jesús De La Rosa; Hernán Batista; Andrés \\ Cantillo; Damiana De La Hoz; Ruth De La Hoz; Germán De La Hoz Bolaño; \\ Amabis Francia; Joel Francia; Merides Hernández; Luz Hernández; \\ Ubaldo Hernández; Cristina Merino; Jadinis Muñoz; Alvaro Ospino; \\ Cilsa Ospino; Rosmy Rangel; Marlith Pérez y Alfonso Pérez Moreno ${ }^{3}$ \\ IED Luz Marina Caballero en el corregimiento de Bellavista-Concordia/Magdalena \\ heiderjose91@gmail.com
}

Para citar este artículo:

De León-Sanabria, H., Jiménez, L., Cervantes, N., De La Rosa, J., Batista, H., Cantillo, A., De La Hoz, D., De La Hoz, R., De La Hoz Bolaño; Amabis Francia; Joel Francia; Merides Hernández; Luz Hernández; Ubaldo Hernández; Cristina Merino; Jadinis Muñoz; Alvaro, G., Ospino, C., Rangel, R., Pérez, P. y Pérez, A. (2018). Fortalecimiento de la conducta pro-ambientales en la IED Luz Marina Caballero. Cultura. Educación y Sociedad 9(3), 325-334. DOI: http://dx.doi.org/10.17981/cultedusoc.9.3.2018.37

\section{Resumen}

La relación del hombre con el ambiente, es inherente a la existencia, por tal motivo la promoción de conductas pro-ambientales se ha convertido en foco atencional en los últimos años, buscando fortalecer acciones sustentables, dentro de los márgenes de la responsabilidad ambiental y social. A continuación, el presente estudio buscó entonces Identificar los componentes pro-ambientales que se pueden fortalecer a través de la investigación para la Formación de Cultura Ciudadana, la Conservación del Medio y el Desarrollo Sostenible en la Comunidad Educativa de la IED Luz Marina Caballero. La investigación fue abordada desde la mirada cuantitativa, con alcance descriptivo. La población abordada fue la comunidad educativa de la IED Luz Marina Caballero. Los resultados permiten ver como las conductas pro-ambientales, se orienta al mantenimiento óptimo de los recursos naturales, al equilibrio del ecosistema y a su vez responde a las necesidades sociales e individuales.

Palabras clave: conductas ambientales, relación hombre-ambiente, responsabilidad social, responsabilidad ambiental, cultura ciudadana, desarrollo sostenible.

\section{Abstract}

The relationship of man with the environment is inherent to existence, for this reason the promotion of pro-environmental behavior has become an attention focus in recent years, seeking to strengthen sustainable actions, within the margins of environmental and social responsibility. Next, the present study seeks to identify the pro-environmental components that can be strengthened through research for Citizen Culture Training, Environmental Conservation and Sustainable Development in the IED Luz Marina Caballero Educational Community. The research will be approached from the quantitative perspective, with descriptive scope. The target population was the educational community of the IED Luz Marina Caballero. The results allow us to see how pro-environmental behaviors are oriented towards the optimal maintenance of natural resources, the balance of the ecosystem and, at the same time, responding to social and individual needs.

Keywords: environmental behaviors, man-environment relationship, social responsibility, environmental responsibility, citizen culture, sustainable development

\footnotetext{
1 Este artículo ha sido derivado del Proyecto Fortalecimiento de la Cultura Ciudadana y Democrática en CT+I a través de la IEP apoyada en TIC en el Dpto. del Magdalena. Ciclón.

2 Líder de investigación del grupo "Los Ambientalistas de Bellavista".

3 Docentes investigadores de la IED Luz Marina Caballero en el corregimiento de Bellavista-Concordia/Magdalena. Grupo de investigación "Los Ambientalistas de Bellavista". Ciclón Magdalena, estrategia N². Universidad De la Costa CUC.
}

- The author; licensee Universidad de la Costa - CUC.

Cultura, Educación y Sociedad vol. 9 no. 3, pp. 325-334. Diciembre, 2018

Barranquilla. ISSN 2389-7724 Online 


\section{Introducción}

Según Berenguer (1998) los estudios en el comportamiento pro ambiental se han desarrollado teniendo en cuenta los componentes motivacionales inmersos en el ser humano. Entre ellos tenemos la valoración hacia el Medio Ambiente en el que se mueve el individuo, el comportamiento altruista y la preocupación por sí mismo y por los beneficios del Medio Ambiente, en el trascurso de los años se han extendido las investigaciones que aluden a los comportamientos ecológicos y las actitudes pro ambientales pues han incrementado las problemáticas en cuanto a contaminación de la tierra y el agua, la adecuada explotación de los recursos que proporciona el planeta, el desenfrenado crecimiento de la industria, entre otros aspectos.

De acuerdo a lo anterior se ha establecido una situación social medioambiental que puede considerarse como crisis, cual está determinada principalmente por la interacción de las personas con la naturaleza y esta problemática se ha producido por falta de conocimiento, pero se debe tener en cuenta que hay variables que afectan al mismo, de manera directa o indirecta tales como: políticos, culturales, económicos y sociales. Por otro lado, es notable que el ser humano con el pasar de los tiempos haya encontrado la manera de interactuar con los demás, mediante emociones y racionalidad, pero este puede variar según la persona y no siempre puede ser muy buena su interacción con la naturaleza. Una gran preocupación con relación a los temas ambientales y su impacto en la crisis global, y es por tal motivo que se debe fortalecer el interés en la búsqueda de estrategias y la promoción de programas que permitan la conservación ambiental, de igual manera se logren modificar ciertas prácticas culturales, en función del consumo sustentable, siendo este resultado de los distintos roles que los seres humanos asumen en su interrelación con la sociedad (Cortés, 2011; Herrera-Tapias, 2014).
Colombia es un país privilegiado por la densidad y cantidad de recursos naturales que posee; es el tercer país más rico en agua a nivel mundial, aproximadamente el $40 \%$ de su territorio está cubierto de bosques, sin embargo, la deforestación, incendio forestales, tala de árboles, contaminación de los ríos, uso descontrolado de productos químicos como insecticidas, aerosoles, la caza y la pesca descontrolada y de más están desatando una reacción en cadena, lo cual está creando situaciones susceptibles de mejora en los medios ambientales, sociales y económicos que también han afectado a nuestra comunidad. Esta problemática genera la necesidad de crear un espacio para la reflexión, conservación, cuidado y preservación de nuestro medio ambiente.

Para abordar un proyecto primero se tiene que tener en cuenta y analizar su necesidad que lo han originado Esta puede ser una problemática o problema de cualquier índole. En este caso estaríamos desarrollando un proyecto de educación ambiental que hace parte del servicio público educativo. En Colombia en los artículos del 78 al 82 del capítulo 3 se contempla el derecho a gozar de un ambiente sano y propicio para el bienestar del hombre y de participar en todas las actividades y decisiones que puedan afectarlo.

"A nivel nacional, los países de América Latina y el Caribe enfrentan numerosos y diversos retos de política ambiental, emanados del desafío común de superar la heterogeneidad estructural y la dependencia en ventajas comparativas estáticas de las industrias intensivas en recursos naturales. La elevada desigualdad y segregación económica de la estructura social, resultante de este camino histórico de desarrollo, condujo a la adopción de patrones de consumo caracterizados por la segregación, con mercados privados de gran desarrollo en el caso de los bienes de alta calidad y, más notablemente, de servicios como el transporte, la salud y la educación. 
Esta segregación separa a los ricos de los pobres, acentúa las disparidades y obstaculiza la puesta en marcha de soluciones públicas más inclusivas y que contemplen la protección del medio ambiente." (CEPAL 2012).

"Actualmente, las ciudades de América Latina y el Caribe se encuentran en un punto de inflexión. Después de décadas en las que los gobiernos centrales y las autoridades locales parecían incapaces de hacer frente a un proceso de cambio demasiado rápido, están dispuestos a trabajar unidos para lograr un desarrollo urbano sostenible. América Latina y el Caribe tienen la posibilidad de salir de los círculos de subdesarrollo, inequidad e insostenibilidad ambiental en los que ha estado inmersa durante décadas e iniciar un nuevo ciclo de transición urbana, para garantizar una mejora fundamental de la calidad de vida en las ciudades" (CEPAL 2012).

Con base en el anterior párrafo, en la Institución Educativa Departamental Luz Marina Caballero, los docentes y estudiantes acompañados por el proyecto ciclón se dieron a la tarea de desarrollar un proyecto de educación ambiental planteando las problemáticas, necesidades, pertinencia y relevancia de la institución y el entorno que lo rodea encaminado a determinar los Comportamientos Pro ambientales que como lo establece Corral-Verdugo, (2000) "Es el conjunto de acciones intencionales, dirigidas y efectivas que responden a requerimientos sociales e individuales que resultan de la protección del medio", por lo cual las problemáticas que se plantea en la institución y el entorno que nos rodea son: primero, las conductas negativas de las personas con respecto al cuidado del medio ambiente (la quema de bosques, la deforestación, tira de basuras en los espacios limpios o naturales, la contaminación de los ríos, ciénagas y lagos).
El desarrollo sostenible como todos sabemos se soporta en tres pilares fundamentales: ecológico, económico y social, los cuales buscan que las acciones que se desarrollen en cualquier sistema o proceso que involucre el territorio logren el equilibrio de los tres pilares, haciendo que las relaciones entre los asuntos ecológicos y sociales sean soportables y que las relaciones entre lo económico y lo ecológico sean viables. Es decir que los procesos de desarrollo económico no alteren los sistemas ambientales y que las relaciones entre los procesos económicos y los sistemas sociales sean equitativas, que los productos económicos se compartan a una buena parte o mayoría de la población (Páez, 2014).

Las problemáticas que se plantea en la institución y el entorno que rodea a los habitantes del corregimiento de Bellavista son: primero, las conductas negativas de las personas con respecto al cuidado del medio ambiente (la quema de bosques, la deforestación, tira de basuras en los espacios limpios o naturales, la contaminación de los ríos, ciénagas y lagos).

En segundo lugar, la caza de animales en vía de extinción \{la hicotea, la higuana el venado, el conejo de monte, el armadillo entre otros). En tercer lugar, la perdida de la flora o plantas de nuestra región En cuarto lugar, con respecto a nuestra institución la mala utilización de nuestros baños, la tira de basura en los pasillos y demás lugares de la institución. La falta de mecanismos o implementos que ayuden a mantener un ambiente sano.

Frente a estas problemáticas surge la necesidad de crear mecanismos para solucionarlos encontrándose casos en los que las estrategias se en encaminado en sensibilizar a las personas por medio de la reflexión, actividades y pedagogía conductas pro ambientales, donde se puedan desarrollar y llevar a cabo acciones que promuevan el cuidado del medio ambiente. 
Cualquier estrategia que propenda al logro de los referidos propósitos implica que los actores educativos participen activamente en el proceso de cambio y transformación social. Al respecto, De la Concepción (2015), concibe los procesos de participación como la vía más expedita para que los individuos desarrollen una pedagogía para la convivencia pacífica y ciudadana.

Por otra parte, son muchos los científicos desde las ciencias sociales y humanas que han enfocado sus esfuerzos en la comprensión de las variables, tanto internas como externas, que favorecen la generación de comportamientos pro ambientales, desde muchas esferas disciplinarias, han buscado ofrecer información importante sobre los motivos que conducen a la mayoría de las personas a generar comportamientos insostenibles, a pesar de la evidencia innegable de la destrucción del planeta y sólo unas pocas, hacen congruentes sus creencias con sus acciones frente a la conservación y protección del Medio Ambiente (CorralVerdugo y De Quiroz, 2004).

En el caso de la región o comunidad promover estrategias para que el estado o sus instituciones, en este caso las alcaldías municipales, ayuden a controlar los actos de deterioro ambiental y sancionar a quienes no cumplan, y a la vez creando mecanismos como un relleno sanitario donde la comunidad pueda tirar las basuras y evitar la contaminación de los ríos, ciénagas, lagos y zonas verde.

En consecuencia, es pertinente o conveniente para un proyecto de educación ambiental relacionar oportunamente sus problemáticas vigentes con los mecanismos de solución como una adecuación y mejoramiento de nuestro espacio, buscando siempre una correspondencia con lo que se quiere lograr, que es la preservación y el cuidado del medio ambiente. Hoy en día los temas sobre el medio ambiente tienen mucha relevancia debido a los cambios climáticos que se están presentando y el deterioro de los ecosistemas por la contaminación, hablar de educación ambiental y conductas pro ambientales, son temáticas actuales de suma importancia tanto a nivel local como a nivel mundial y que a diferencia de otros temas sobresalen por su impacto en nuestro entorno físico.

Por lo tanto, como expresan Chumaceiro, Hernández y Chirinos (2016), es necesario educar ciudadanos conscientes de sus derechos y de sus deberes ecológicos a través de la consolidación de valores ético, del compromiso social y ambiental como paradigma general, transmitiendo conocimientos y desarrollando habilidades que se ajusten a estos requerimientos

Pues es urgente crear espacios para la reflexión y preservación del medio ambiente, debido a los sucesos que se están presentando en nuestras sociedades y en los ecosistemas sobre el problema de la contaminación en sus diferentes grados y formas que termina por acabar con nuestros recursos naturales.

Desde la línea de Investigación Problemáticas en las que se encuentran los maestros y maestras, además de la línea de Medio Ambiente se pretende obtener evidencias empíricas concluyendo en aportes significativos basados en los comportamientos pro-ambientales, los cuales a manera de diagnóstico que permitirían la caracterización de las actitudes y comportamientos de los estudiantes hacia el fortalecimiento de las competencias ciudadanas y la inserción de la investigación a las practicas pedagógicas.

Por tal razón, el abordaje investigativo implica necesariamente el establecimiento de objetivos pertinentes e incluir un modelo teórico de explicación del comportamiento pro ambiental basado en la comprensión del desarrollo de competencias.

La naturaleza puede ser explicada como lo que existe y se reproduce, inherentemente de la actividad humana, este puede ser dinámico, cambiante y adaptable. El ser 
humano, sin importar su nivel de complejidad en lo relacionado con sus condiciones, existe en una naturaleza que afecta y de la que se ve afectado. Los individuos habitan en las esferas tanto históricas como naturales, "Como ser histórico, y por tanto como ser social, humaniza a la naturaleza, pero también la conoce y reconoce como totalidad absoluta, como causa que se basta a sí misma, como condición y supuesto de la humanización” (Kosik, 1967)

\section{Comportamientos Pro Ambientales}

Hasta finales del Siglo XX, se hablaba de una conducta pro-ecológica, definida como "El conjunto de acciones efectivas y deliberadas que resultan en la protección de los recursos naturales o, por lo menos, en la reducción del deterioro ambiental" (Corral, 2001); entendiendo que el ambiente al que se referían estaba relacionado específicamente con la naturaleza, dejando de lado el componente social para su estudio. Se reconocían las normas, valores sociales como inductores de comportamientos proambientales de manera explícita, sin embargo, eran estudiados de manera aislada, a través de la Psicología de Conservación del ambiente (Corral-Verdugo, 2010).

Sin embargo, las limitaciones conceptuales y metodológicas para el estudio de la "Conducta proecológica" poco a poco fueron llevando a la comunidad científica al planteamiento de nuevas ideas en las que se tuviera en cuenta, no sólo un enfoque de investigación basado en lo correctivo, sino también una definición más inclusiva del componente individual y social, en el que se pueda reconocer un escenario físico que se debe cuidar y una proyección hacia el futuro mediante las acciones de los individuos en sus contextos específicos de desarrollo (Herrera, 2011).

Las definiciones empleadas para designar los comportamientos relacionados con el cuidado del Medio Ambiente incluyen, entre otros, términos como comportamiento ambiental, conducta ecológica, conducta ecológica responsable, conducta sustentable; que son coherentes con la diversidad de enfoques desde donde se hacen aproximaciones teóricas, metodológicas y prácticas (Martínez-Soto, 2006), sin embargo, para efectos de la presente investigación tomamos como referencia el concepto de "Comportamiento pro ambiental".

El Comportamiento Pro ambiental "Es el conjunto de acciones intencionales, dirigidas y efectivas que responden a requerimientos sociales e individuales que resultan de la protección del medio" (CorralVerdugo, 2000). Tomando como referencia tales aportes y los de Pozo (2006) se identificaron elementos teóricos que, bajo los distintos enfoques de la psicología, buscando identificar aspectos de explicación y generación del comportamiento pro-ambiental. Se presentará para el análisis del comportamiento pro ambiental, orientado a las actitudes, el desarrollo de competencias en los individuos, en las relaciones sociales y con el medio para la conservación, los componentes psicológicos y las interacciones.

\section{Comportamientos Pro ambientales y Educación}

De acuerdo a la establecido por el MEN (2005) al hablar de desarrollo sostenible y de lo relevante que es el trabajo de las temáticas transversales para "la mejora de la calidad educativa; la dimensión ética, cultural y científica de la educación ambiental; la acción interinstitucional da vida y continuidad a los procesos; una política nacional que consolida líneas de acción en pro de una educación para un ambiente sostenible." (p.p 35). Al respecto, Mujica, Marín, Smith y Lovera (2008), destacan que la sosteniblidad del desarrollo estará asociada a iniciativas locales que se fundamentan en el emprendimiento y las relaciones entre los actores sociales. 
"Un componente importante implícito en todas las definiciones de desarrollo sustentable se relaciona con la equidad: la equidad para las generaciones por venir, cuyos intereses no están representados en los análisis económicos estándares ni en las fuerzas que desestiman el futuro, y la equidad para la gente que vive actualmente, que no tiene un acceso igual a los recursos naturales o a los bienes sociales y económicos" (Toledo, 1997; MEN, 2005)

Según Centro Virtual de Noticias de Educación (2005) En cuanto a los sistemas educativos latinoamericanos, han sufrido el impacto causado por los cambios puestos a prueba en el planeta. Cambios que producen consecuencias desfavorables y ocacionan el desplazamiento forzoso y, en ocasiones, aculturadores, y asi mismo la apertura hacia procesos de innovación que permitan el ingreso en tales sistemas a los problemas reales de las sociedades (entre ellos, de forma prioritaria, el problema ambiental), con trabajos adaptables al entorno que permitan identificar las necesidades culturales y lingüísticas de cada grupo humano que aprende.

Desde esta perspectiva, los Ministerios de Educación Nacional y de Ambiente, Vivienda y Desarrollo Territorial en Colombia, adelantan estrategias para la inclusión de la dimensión ambiental en la educación formal - a partir de las políticas nacionales educativa y ambiental, y la formación de una cultura ética en el manejo del ambiente, mediante la definición y puesta en marcha de los Proyectos Ambientales Escolares; dando asi un alto grado de prioridad al medio ambiente, propiciando el sentido de pertencia y una cultura con responsabilidad ambiental desde la etapa educativa.

Si se pretende efectuar un impacto positivo hacia el medio ambiente, se debe contar con el conocimiento necesario y asi mismo implementar programas acordes con estas necesidades, para posteriormen- te brindarles las respectivas alternativas de solución. "Los PRAES son proyectos pedagógicos que promueven el análisis y la comprensión de los problemas y las potencialidades ambientales - locales, regionales y nacionales-, y generan espacios de participación para implementar soluciones acordes con las dinámicas naturales y socioculturales, donde los docentes juegan un papel fundamental como dinamizadores de este proceso desde la escuela, que son espacios propicios de autorregulación de comportamientos ciudadanos, requeridos para la sostenibilidad del ambiente." (UNICEF. s.f).

El PRAE es considerado dentro de la Política Nacional de Educación Ambiental como una de las estrategias para la inclusión de la dimensión ambiental en los currículos de la educación preescolar, básica y media. Dimensión que debe incluirse a partir de proyectos y actividades y no por medio de una cátedra, de modo que permita integrar las diversas áreas del conocimiento para el manejo de un universo conceptual aplicado a la solución de problemas, llevando esto a la realidad fáctica, es de importante que las actividades y medios que se dispongan para efectuar lo propuesto, se debe ejecutar en un contexto que permita idealizar las situaciones del ambiente tratado, deben ser didácticas y practicas para su fácil entendimiento, lo cual permitirá una mayor interiorización conceptual y aumento de generación de ideas para tratar la problemática.

"Así mismo, permite explorar cual es la participación de cada una de las disciplinas en un trabajo inter o trans-disciplinario, posibilitando la formación en la ciencia, la técnica y la tecnología desde un marco social que sirva como referente de identidad del individuo y genere en compromiso con él mismo y con la comunidad. Adicional a lo anterior, los proyectos escolares en educación ambiental deben estar inscritos en la problemática ambiental local, regional 
o nacional y concertarse con las entidades que de una u otra manera estén comprometidas en la búsqueda de soluciones." (CEPIS 2001); el tema del medio ambiente no da espera y debe ser tratado con brevedad, asi mismo debe ser tenido en cuenta como un pilar fundamental en la educación, no importando la disciplina se debe incentivar y propiciar la responsabilidad ambiental.

\section{Metodología}

La postura epistemológica, la cual sustentó el proceso investigativo es el paradigma positivista, pues permitió la caracterización al hacer la descripción, medición y generalización de los componentes causales del fenómeno, además se caracteriza por ser de tipo aplicada. De igual manera el paradigma brinda las técnicas y las bases teóricas que permiten ser cuantificados y generalizados en los procesos habituales dentro de las actividades académicas. La investigación es de tipo cuantitativa, de corte descriptivo.

\section{Población y muestra}

La población la conformaron los estudiantes del grado $6^{\circ}$ de bachillerato de la IED Luz Marina Caballero, cuyas edades oscilan entre los 10 y 18 años, habitantes del corregimiento de Bellavista en el Municipio de Concordia Magdalena.

\section{Técnicas e instrumentos de recolección de la información}

Para la recolección de la información del estudio se diseñó dos cuestionarios escala tipo Likert. Como forma de recolección de la información fue de tipo cerrada, la primera con 5 opciones de respuesta con 30 ítems pertinente para la identificación de los objetivos abordados en el estudio. Se evaluaron con estas las habilidades en los estudiantes de $6^{\circ}$ de la IED San Juan Bautista pertenecientes al Programa Ciclón.

\section{Procedimiento}

Fase (1): Identificación de la problemática a estudiar.

Fase (2): Definición de la población y muestra.

Fase (3): Definición de las variables de estudio.

Fase (4): Fundamentación teórica.

Fase (5): Escogencia de las técnicas e instrumentos de recolección de información. Fase (6): Aplicación de los instrumentos. Fase (7): Análisis y procesamiento de los datos.

Fase (8): Generación de resultados.

Fase (9): Socialización y discusión de los hallazgos encontrados.

\section{Resultados}

Como resultados del estudio se pudo observar lo siguiente:

Con relación a la primera pregunta sobre la preocupación por los tremas ambientales el $40 \%$ de los encuestados siempre se preocupa por el medio ambiente que los rodea, el $57 \%$ Algunas veces se preocupa, por último, la minoría que corresponde al 3\% de los encuestados Nunca se preocupa por los problemas ambientales de su contexto.

Frente a la responsabilidad sobre los problemas ambientales que ocurren a diario en el contexto, la comunidad educativa manifiesta en un $50 \%$ si se preocupa, un $47 \%$ que algunas veces, mientras que el $3 \%$ dijo no serlo.

De acuerdo a la pregunta sobre la utilización de los basureros públicos para tirar los papeles $44 \%$ respondió Siempre, el $26 \%$ que algunas veces y el $30 \%$ Nunca ha hecho uso de éstos.

En el cuarto interrogante cuando se les preguntó por el uso de los contenedores públicos para depositar de forma separada los desechos el $36 \%$ de los encuestados dijo que Siempre los utiliza, por su parte un $16 \%$ dijo que algunas veces y un $48 \%$ nunca los ha usado. 
Cuando se les preguntó si se ocupaban de darles nuevos usos a las cosas que podría ser útiles para ellos u otras personas el $40 \%$ respondió que Siempre lo hace, el $30 \%$ algunas veces lo hace mientras que el $30 \%$ nunca lo hace.

Con base a la separación de la basura según su tipo, (vidrio, plástico, papel, orgánica u otra) el $20 \%$ dijo haberlo hecho Siempre, el $30 \%$ algunas veces y el $50 \%$ Nunca lo ha hecho.

Frente a la pregunta relacionada con la puesta en práctica de medidas domésticas para economizar el agua el $60 \%$ de la población encuestada Siempre pone en práctica medidas domésticas para economizar agua, por su parte un $10 \%$ lo ha hecho alguna vez y un $30 \%$ nunca lo ha hecho.

Referente a la pregunta sobre el uso sistema de ahorro de energía en el hogar como (ampolletas de bajo consumo, desenchufo de artefactos en desusos, etc.) Un $10 \%$ respondió que si, un $40 \%$ respondió Algunas veces y un $50 \%$ dijo no hacerlo Nunca.

De acuerdo a la pregunta relacionada con la participación en acciones a favor del medio ambiente en lugares (limpieza de playa, parques, plantar árboles, etc.) Un $20 \%$ si lo ha hecho, un $10 \%$ algunas veces y un $70 \%$ nunca ha hecho parte de estas actividades.

En cuanto a la participación en movilizaciones a favor del cuidado del medio ambiente la mayoría, con un 37\% respondió que sí, un 33\% respondió que algunas veces, mientras que un $30 \%$ Nunca ha ido a estas marchas.

Frente a la pregunta relacionada con la preferencia hacia los productos marcados con envases reciclables o reutilizables el $30 \%$ de los encuestados afirma que si, otro $30 \%$ afirma que algunas veces y el $40 \%$ no prefiere productos con envases reciclables o reutilizables.

En el interrogante que se refiere a la compra y preferencia de productos que tengan menor cantidad de envases o empaque- tados por parte de la población un 5\% si compra, un $15 \%$ algunas veces y finalmente un $80 \%$ no compra.

\section{Conclusión}

De acuerdo a lo anterior se puede establecer que como conductas pro-ambientales en la IED Luz Marina Caballero, se orienta al mantenimiento óptimo de los recursos naturales, al equilibrio del ecosistema y a su vez responde a las necesidades sociales e individuales.

Para hacer un buen fortalecimiento de los comportamientos y la conservación de la cultura ciudadana debemos concientizar a la comunidad educativa y en general de la institución educativa Luz Marina Caballero, de la conservación del medio ambiente, el adecuado manejo y uso de los desechos orgánicos e inorgánicos, hablar con las entidades gubernamentales, para que en conjunto con la institución se propongan alternativas que promuevan capacitar y concientizar para que se haga un adecuado uso de los recursos que posee el medio en el que se interactúa en sus diferentes formas organizando campañas de aseo en las calles y patios de la población con limpiezas y ornamentación en la plaza, lugares deportivos.

Por lo tanto, la complejidad de la problemática ambiental no solo implica aprender nuevos hechos, sino que abre una nueva perspectiva en todas las disciplinas incluyendo la pedagógica y la psicológica permitiendo una nueva racionalidad en el individuo que le permite reapropiarse del conocimiento desde el ser del mundo y del ser humano en el mundo del existencialismo distinguiendo el saber y la identidad incorporándose el ser de cada individuo y cada cultura. Otro aporte significativo para fortalecer las conductas pro-ambientales que contribuyan a la formación ciudadana, la conservación del medio y el desarrollo sostenible en la comunidad educativa que habla del comportamiento pro-ambiental. 
Una aproximación al estudio del desarrollo sostenible comportamiento persona-ambiente. (Sauvé, 1999; Novo, 2009).

\section{Referencias}

Amérigo, M. y González, A. (1996). Preocupación medioambiental en una población escolar. Revista de Psicología Social Aplicada, 6. 75-92.

Aula planeta. (04 de 11 de 2015). Recursos para la creación de muros digitales. Recuperado de www.aulaplaneta.com: http://www.aulaplaneta.com/2G15/ 1l/04/recursos-tic/cincoherramientaspara-crear-murales-digitales/

Berenguer, J. (1998). Actitudes y creencias ambientales. Una explicación psicosocial del comportamiento ecológico. [Tesis doctoral]. Universidad Autónoma de Madrid, Madrid.

CVNE. (2005). La importancia de las experiencias en Educación Ambiental. Centro Virtual de Noticias de Educación. Instituciones Universitarias, Corporación Universitaria de la Costa, CUC. Recuperado de http://www.mineducacion.gov.co/cvn/1665/article-91671. html

Chumaceiro H., Hernández G. y Chirinos N. (2016). Responsabilidad social universitaria, desarrollo sostenible y ciudadanía ambiental. Cuadernos de RSO, 4 (1). 53 - 64 .

Corral-Verdugo, V. (2000). La definición del Comportamiento proambiental. La psicología social en México, 8(1). 466-467.

Corral-Verdugo. (2010). Psicología de la sustentabilidad. Un análisis de lo que nos hace pro ecológicos y pro sociales. México, D.F.: Trillas.

Corral-Verdugo, V., y De Queiroz, J. (2004) Aproximaciones al estudio de la conducta sustentable. Medio Ambiente y Comportamiento Humano, 5(1 y 2). 1-26.
Cortez, O. (2011). Comportamiento pro ambiental y pensamiento económico en la construcción del desarrollo sostenible. Cultura, educación y sociedad, 2(1). 4356.

De la Concepción, A. (2015). Concepciones sobre participación de niñas, niños y adolescentes: Su importancia en la construcción de la convivencia escolar. Cultura Educación y Sociedad 6(2). Recuperado de https://revistascientificas.cuc.edu.co/culturaeducacionysocie$\mathrm{dad} /$ article/view/840

Darley, J. y Beniger, J. (1981). Diffusion of energy conserving innovations. Journal of Social Issues, 37(2), 150-171.

CEPIS. (2001). Marco conceptual de los residuos sólidos. Recuperado de http:// www.bvsde.paho.org/bvsars/e/fulltext/ marco/marco.pdf

UNICEF. (s.f). Experiencias urbanas de gestión integral de residuos en 10 municipios de Argentina. Recuperado de https://www.unicef.org/argentina/spanish/EcoclubesbajaWEB.pdf

González, A. y Amérigo, M. (1999). Actitudes hacia el Medio Ambiente y conducta ecológica. Psicothema, 11(1), 13-25.

Herrera, K. (2011). La crisis medioambiental. Una mirada desde la Psicología. Barranquilla: Educosta.

Herrera-Tapias, B . (2013). La constitucionalización de los derechos del consumidor en Colombia: un análisis desde los derechos sociales fundamentales. $R e$ vista civilizar ciencias sociales y humanas. 13(25).

Kosik, K. (1967). Dialéctica de lo Concreto. México, D.F.: Editorial Grijalbo.

Martínez-Soto, J. (2006) Comportamiento proambiental. Una aproximación al estudio del desarrollo sustentable con énfasis en el comportamiento persona-ambiente. Argentina: Red Theomai. 
MEN. (2005). Educar para el Desarrollo Sostenible. Altablero 36. Recuperado de http://www.mineducacion.gov.co/1621/ article-90893.html

Mujica de López, M., Marín, F., Smith, H., Lovera, M. (2008). Municipio innovador, un modelo para el desarrollo local sostenible. Multiciencias, 8 [en linea] Disponible en <http://www.redalyc.org/articulo. oa?id=90411691019 $>$ ISSN 1317-2255

Suárez, E. (1996). La participación ambiental como conducta altruista. En Ciudad y medio ambiente desde la experiencia humana. Mongrafies Psico/Socio/Ambientals, 10. 289-294.

Sauvé, L. (1999). La educación ambiental entre la modernidad y la posmodernidad: En busca de un marco educativo de referencia integrador. Tópicos, 1(2). Recuperado de http://www.ecominga.uqam.ca/ PDF/BIBLIOGRAPHIE/GUIDE_LECTURE_1/5/2.Sauve.pdf
Novo, M. (2009). La educación ambiental, una genuina educación para el desarrollo sostenible. Revista de Educación. Universidad Nacional de Educación a Distancia (UNED). Madrid, España. Recuperado de: http:// www.revistaeducacion.mec.es/re2009/ re2009_09.pdf

PNUMA. (1990). Educación ambiental: modulo para la formación de profesores de ciencias y de supervisores para escuelas secundarias. Orelac. Recuperado de http://unesdoc.unesco.org/ images/0007/000714/071480so.pdf 